\title{
Cutaneous Melanoma by AJCC v8 Stage
}

National Cancer Institute

\section{Source}

National Cancer Institute. Cutaneous Melanoma by A/CC v8 Stage. NCI Thesaurus. Code C137645.

A term that refers to the staging of cutaneous melanoma, following the rules of the TNM AJCC v8 classification system. This staging system does not apply to melanoma of the conjunctiva, melanoma of the uvea, mucosal melanoma arising in the head and neck, and mucosal melanoma of the urethra, vagina, rectum, and anus. (from AJCC 8th Ed.) 